Low noise DC to DC converters for the sLHC experiments

This article has been downloaded from IOPscience. Please scroll down to see the full text article. 2010 JINST 5 C11011

(http://iopscience.iop.org/1748-0221/5/11/C11011)

View the table of contents for this issue, or go to the journal homepage for more

Download details:

IP Address: 137.138.124.142

The article was downloaded on 11/07/2011 at 09:56

Please note that terms and conditions apply. 
Topical Workshop on Electronics for Particle Physics 2010, 20-24 SEPTEMBER 2010, AACHEN, GERMANY

\title{
Low noise DC to DC converters for the sLHC experiments
}

\section{B. Allongue,${ }^{a}$ G. Blanchot,,${ }^{a, 1}$ F. Faccio, ${ }^{a}$ C. Fuentes, ${ }^{a, b, 2}$ S. Michelis ${ }^{a, c}$ and S. Orlandi ${ }^{a}$}

\author{
${ }^{a}$ CERN, \\ Route de Meyrin, CH-1211 Geneva 23, Switzerland \\ ${ }^{b}$ Department of Electronics, UTFSM, \\ Avenida España 1680, Valparaiso, Chile \\ ${ }^{c}$ Electronics Laboratory, Institute of Electrical Engineering, EPFL, \\ ELB111, Station 11, CH-1015 Lausanne, Switzerland \\ E-mail: georges.blanchot@cern.ch
}

\begin{abstract}
The development of front-end systems for the ATLAS tracker at the sLHC is now in progress and the availability of radiation tolerant buck converter ASICs enables the implementation of DC to DC converter based powering schemes. The front-end systems powered in this manner will be exposed to the radiated and conducted noise emitted by the converters. The electromagnetic compatibility between DC to DC converters and ATLAS short strip tracker hybrid prototypes has been studied with specific susceptibility tests. Different DC to DC converter prototypes have been designed following a noise optimization methodology to match the noise requirements of these front-end systems. The DC to DC converter developed in this manner presents a negligible emission of noise that was confirmed by system tests on an ATLAS tracker front-end module prototype. As a result of this, power converters can now be integrated in close vicinity of front-end chips without compromising their overall noise performance.
\end{abstract}

KEYWORDS: Voltage distributions; Analogue electronic circuits; Radiation-hard electronics; Frontend electronics for detector readout

\footnotetext{
${ }^{1}$ Corresponding author.

${ }^{2}$ Cristian Fuentes is supported by MECESUP-Chile under grant FSM0601.
} 


\section{Contents}

1 Introduction 1

2 DC to DC converters $\quad 2$

2.1 Technological choices 2

2.2 Control of noise sources 3

2.3 Prototypes 5

2.3.1 SM01B converter 5

2.3.2 AMIS2EMC converter 5

3 System tests 6

3.1 Susceptibility properties of an ATLAS tracker module 6

3.2 Compatibility of the AMIS2 and SM01B converters with an ATLAS tracker module 7

4 Conclusions 9

\section{Introduction}

Today's high energy physics experiments at LHC embed large and very sensitive front-end electronics systems that are usually remotely powered through long cables. The innermost region of the experiments, the trackers, are those providing the largest density of channels, that must be powered with the minimal mass of cables and with reduced heat dissipation to avoid complex and massive cooling systems.

With the upgrade of the accelerator and its physics experiments already being planned, the detectors will require an increased number of electronic readout channels, which will demand more power. This increase of delivered power should be achieved without the addition of material in the detector volume, because of lack of physical space to run more cables and because material in this volume is detrimental to the physics performance of the detector.

Two solutions to deliver more power without increasing the cable volume and mass are under study, one is based on the powering of serially interconnected modules [1], and the other relies on the distribution of power through on-detector DC-DC converters [2]. These converters must provide a reliable operation in high radiation (total ionizing dose up to $250 \mathrm{Mrad}(\mathrm{SiO} 2)$ and neutron fluencies up to $2.5 \times 10^{15} \mathrm{n} / \mathrm{cm}^{2}, 1 \mathrm{MeV}$ neutron equivalent, based on the simulated environment in the central tracker detector over its projected lifetime) and strong DC magnetic field environment (up to $4 \mathrm{~T}$ ) of the detector.

A comparative study indicated that the buck converter is one of the most suitable converter topology for the intended application [3]. Given the range of available coreless inductors, the switching frequency has to be set beyond $1 \mathrm{MHz}$ in order to limit the current ripple. 


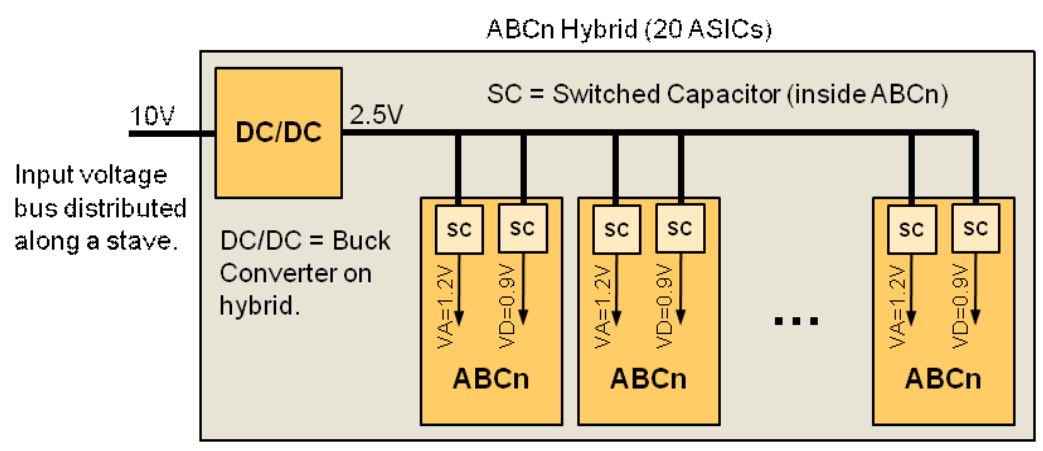

Figure 1. Powering scheme based on DC/DC converters proposed for the ATLAS upgraded tracker.

A typical tracker front-end system is made of strip detectors that are bonded to front-end hybrid circuits. These hybrids are fitted with several front-end chips. Several hybrid and detector modules are then mounted together to form a stave or a supermodule [4, 5]. Based on this and on the estimated power requirements of the hybrids, an optimal powering scheme based on DC-DC converters has been proposed for ATLAS [2], that relies on an input voltage bus (10V) distributed along the stave to all the hybrids. Each hybrid circuit would be equipped with one Buck DC/DC converter delivering an intermediate bus voltage $(2.5 \mathrm{~V})$ that brings the power to each front-end chip with a conversion efficiency of $80 \%$. Each front-end chip would then convert the intermediate voltage down to the levels that it requires $(1.2 \mathrm{~V}$ and $0.9 \mathrm{~V})$ through integrated point-of-load switched capacitors DC/DC converters, whose efficiency is expected to be around 95\% [6]. The need for switched capacitor converters depends on the technology used for the front-end ASICs. This paper presents the development of two noise optimized buck converters for tracker applications, one using a radiation tolerant $\mathrm{ASIC}$ delivering up to $2 \mathrm{~A}$ at $2.5 \mathrm{~V}$ output voltage, and the other using a commercially available controller delivering up to $5 \mathrm{~A}$ with the same output voltage. The latter is suitable for currently available ATLAS tracker front-end systems based on the ABCN ASIC in $0.25 \mu$ CMOS technology [7], and the radiation tolerant counterpart targets systems implemented in a $0.13 \mu$ technology not yet available.

\section{DC to DC converters}

\subsection{Technological choices}

Different DC to DC conversion technologies applicable to the trackers at sLHC have been studied in detail. In particular, the impossibility of using ferromagnetic materials for the inductors in the large magnetic field in SLHC experiments, together with the low-volume and low-mass requirements for the converter, imposed strict boundaries to the usable technologies. The Buck converter topology was selected because of its low parts count, with the possibility to integrate most of it in an ASIC, except the main coil.

The required tolerance to high levels of radiation has driven the qualification process of several microelectronic technologies [8]. Amongst those, a $0.35 \mu \mathrm{m}$ technology was fully qualified and ASIC prototypes have been designed and produced, the latest one being the AMIS2 ASIC [9]. It integrates the full control loop, the bandgap reference voltage generation, and a relatively simple 

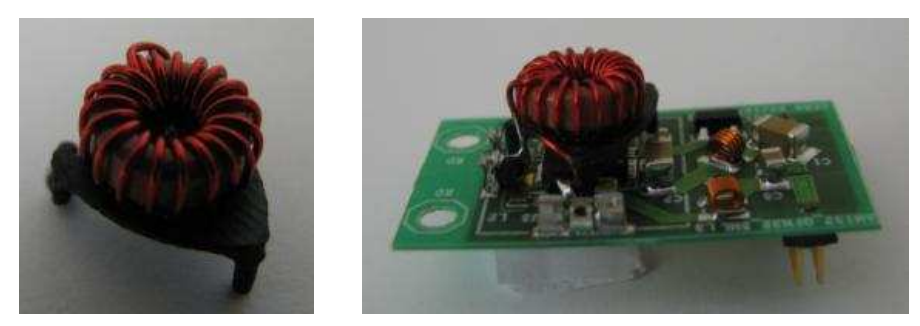

Figure 2. Custom made Coilcraft inductor (left) mounted on top of an AMIS2 converter (right).

handling of the dead times. This prototype still required an off-chip voltage regulator to provide the low-voltage $(3.3 \mathrm{~V})$ powering the on-chip control logic and did not include any protection feature yet. The performance of the AMIS2 prototype is good, and the converter turned out to be very robust to radiation effects. An efficiency of 75-80\% was measured for samples mounted in QFN32 packages, for a regulated output voltage of $2.5 \mathrm{~V}$, input voltage of $10 \mathrm{~V}$ and output current of $1 \mathrm{~A}$.

To be compatible with the tracker environment the proposed buck converter requires a low mass, low resistance air core power inductor to deliver a DC output voltage. Electromagnetic simulations and measurements showed that the toroidal topology allows containing the emission of magnetic field significantly, compared with the solenoidal topology. Flat PCB toroidal inductors were evaluated initially: the difficulty to reliably embed low resistance vias forced us to withdraw this technique, in favor of handmade toroidal coils using insulated copper wires. The resulting compact geometry was found to be favorable to reduce the magnetic field emissions; placed on top of the converter ASIC the board area is minimized. The manufacturability of this coil is a critical issue and recent contacts with industry resulted in the development of a custom compact air core toroidal inductor of $200 \mathrm{nH}$ that matches the converters requirements (figure 2).

The strong susceptibility of front-end systems to electric fields was put in evidence through system tests made with the early prototype converters [6]. Despite the significant improvement in terms of noise mitigation by proper layout, the radiated fields can't be fully cancelled. The addition of a shield enclosing the noise emitting parts and board areas was found to be a very effective way to enable the placement of the converters in the close vicinity of the sensitive front-end circuits. Copper foils of $200 \mu \mathrm{m}$ thickness have been successfully used for handmade shield assemblies; the copper coating of molded plastic structures is under study now for a manufacturable and low mass shielding solution.

\subsection{Control of noise sources}

If the addition of a shield on top of the converter certainly helps in reducing the electromagnetic coupling with the front-end system, it still must be considered as an option that comes only after having applied good design practices to limit the emission of noise by layout and placement of parts. The identification of the dominant sources of noise in a buck converter is represented in figure 3. The major source of electric field is found at the phase node (N3) that carries a square signal of input voltage amplitude at the switching frequency of the converter. The area of this node on the board is minimized but limited to the connectivity needs of the converter ASIC, hence it can't be fully cancelled and the use of shield is here essential. The other dominant sources emit magnetic field. The main inductor (L) carries a large triangular current along loop A with peak 


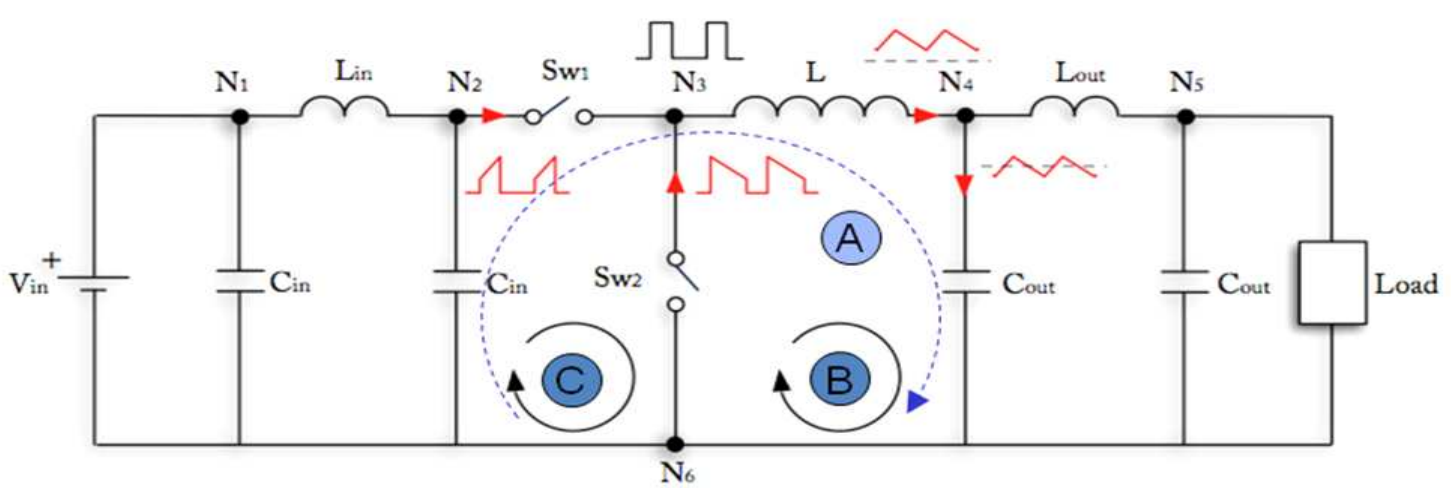

Figure 3. Noise sources in a buck converter.

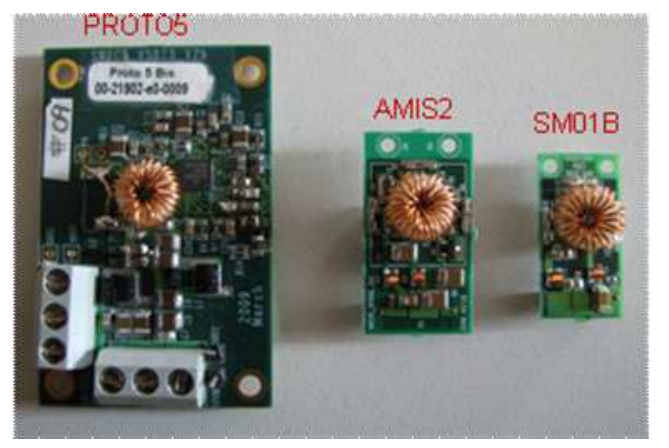

Figure 4. The commercial controller based Proto5 converter compared with the new AMIS2 and SM01B plug-in converters.

to peak amplitudes of several Amperes that radiate mainly at the switching frequency along the circular path of the toroidal structure. This current is sliced by the action of the switches SW1 and SW2 resulting in two trapezoidal current waveforms with fast current transitions along loops A (ON time) and B (OFF time) that are the dominant source of magnetic field in the high frequency domain. The transition between $\mathrm{ON}$ and $\mathrm{OFF}$ times results in fast transient impulses along loop $\mathrm{C}$ contributing also with magnetic emissions in the high frequency domain.

The mitigation of the radiated fields is initially achieved with the reduction of the loop areas. This is obtained at board level by putting the input and output decoupling capacitors as close as possible to the switching ASIC. The addition of the input and output $\pi$ filters reinforces this effect blocking the emission of conducted noise along the input and output ports.

The parasitic parameters of selected passive components are known to impact strongly on the conducted emissions in the form of common mode and differential mode noise [10]. Recent studies allowed putting in evidence the impact of magnetic coupling effects between the components that carry large currents and those that carry filtered currents. The segregation of noisy and quiet parts at the board level, reinforced with the addition of the shield between them, resulted in a very effective way to directly mitigate the radiated noise, indirectly mitigating the conducted emissions too. 

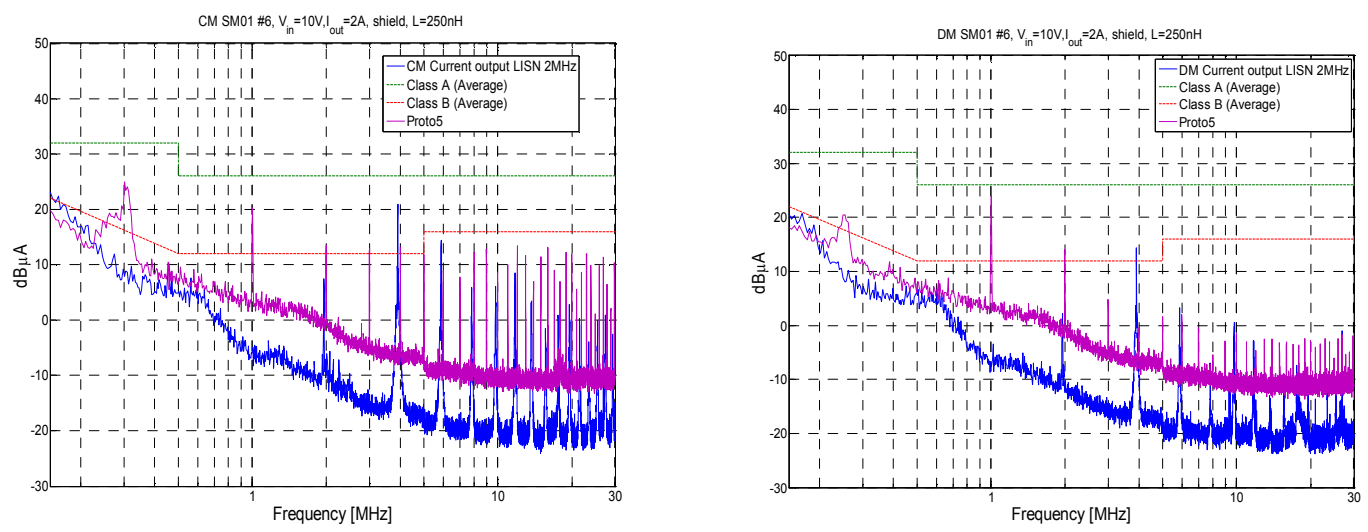

Figure 5. Common Mode (left) and Differential Mode (right) emissions of the SM01B prototype (in blue) compared to those of Proto5 (in pink) and to the IEC-CISPR11 Classes A and B standard limits.

\subsection{Prototypes}

Two converters have been produced applying the mitigation techniques described earlier, namely the SM01B converter that used a commercial buck controller, and the AMIS2EMC converter designed on the basis of the AMIS2 ASIC with a particular emphasis on the control of magnetic coupling effects at board level. These converters have a typical size of $26 \mathrm{~mm} \times 13 \mathrm{~mm}$, and are much more compact than the earlier Proto5 prototype (figure 4) used to compare their performances [6].

\subsubsection{SM01B converter}

To enable the tests on systems using the ABCN25 ASICs in $0.25 \mu \mathrm{m}$ CMOS technology, a DC to DC prototype module was designed using the Linear Technology LT3605 buck controller: this commercially available device offers a topology very similar to the one of AMIS2 ASIC, excepting the bootstrap circuitry that remains external and imposes a multilayer layout. This module, identified as SM01B (figure 4), delivers $2.5 \mathrm{~V}$ with an output current of up to 5A. Its conducted noise is compared with the one from the Proto5 prototype converter, with an improvement of more than 10 $\mathrm{dB}$ in the frequency range comprised between $10 \mathrm{MHz}$ and $30 \mathrm{MHz}$ where the front-end systems are most sensitive (figure 5).

\subsubsection{AMIS2EMC converter}

Once the first functional radiation tolerant AMIS2 ASIC was delivered, a prototype board was designed for it (figure 4). The AMIS2 ASIC includes the power transistors and requires a reduced amount of passive components. Packaged in a QFN48 plastic case, a maximum common mode noise current of $20 \mathrm{~dB} \mu \mathrm{A}$ was obtained, which is comparable to the level achieved with the earlier Proto5 prototype (figure 4, based on the Intersil ISL6540A controller). System tests revealed however some susceptibility to radiated noise from the converter, and this motivated a new design that minimized the magnetic couplings and introduced the shield as described in section 2.2. With this new level of optimization and understanding, the AMIS2EMC prototype using the same AMIS2 ASIC but in a more compact QFN32 package was produced (figure 4). The level of noise achieved is outstanding at $-5 \mathrm{~dB} \mu \mathrm{A}$, a level never observed so far in any converter tested at CERN (figure 6). 

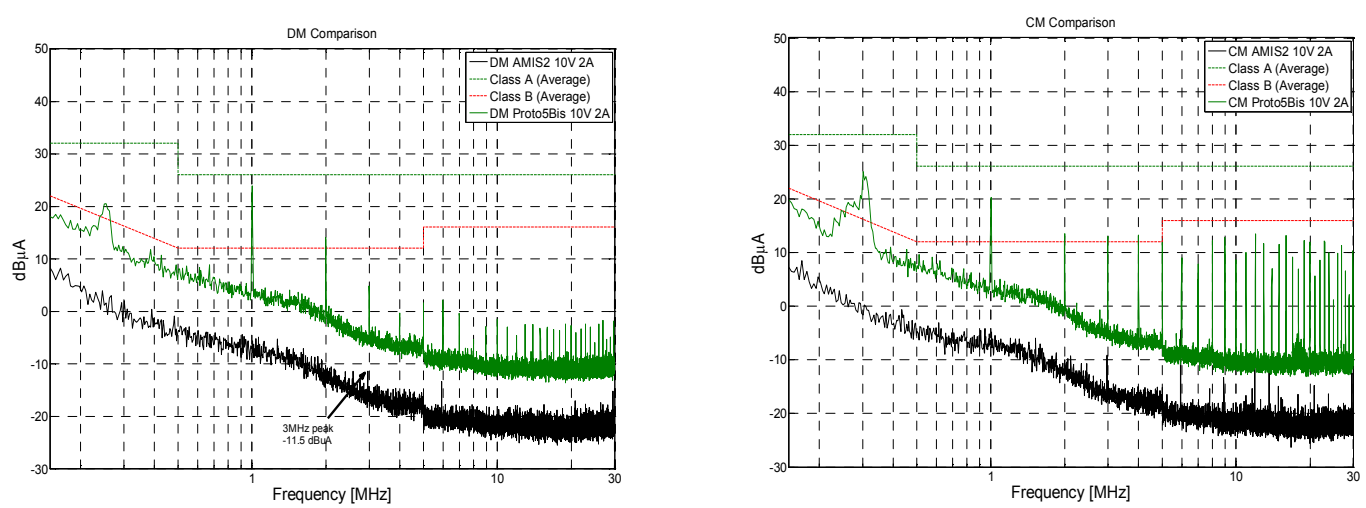

Figure 6. Common Mode (left) and Differential Mode (right) emissions of the AMIS2EMC prototype (in black) compared to those of Proto5 (in green).

\section{System tests}

The noise performance of a system results from its susceptibility with respect to the noise emitted by the converter used to power it. The compatibility between both, in the absence of quantified limits applicable to the noise of the converters, is obtained by means of systems tests. Two ATLAS tracker front-end systems (from the University of Liverpool [5] and from the University of Geneva [4], respectively) have been used to evaluate their compatibility with the DC to DC converters produced. Although both are using the same ABCN front-end ASICs in $0.25 \mu \mathrm{m}$ CMOS technology, their assembly onto hybrids, modules and detectors follow different technical implementations. Each hybrid hosts 20 ASICs arranged in two rows, each ASIC handling 128 strip channels. The input signals are amplified and discriminated to deliver a binary output for every channel. The noise properties of the channels are obtained with the measurement of the output efficiency when varying the discriminating threshold, obtaining a typical error function curve so called S-Curve. Each curve is fitted and its slope is representative of the noise of that channel.

\subsection{Susceptibility properties of an ATLAS tracker module}

The setup at Liverpool was initially used to explore the compatibility with the Proto5 converter. Although it appeared to be insensitive to the common mode and differential mode currents emitted by the converter, the front-end noise increased very significantly once the converter got placed very close to it. Two effects were then observed (figure 7): first the noise of all the channels was globally increased very significantly, and secondly odd channels close to the converter were developing larger noise than even channels.

The global increase of the noise level for all channels independently of their distance to the converter is attributed to magnetic field coupling in the hybrid board. This was later on put in evidence using a near field magnetic loop probe radiating towards the system, with all channels increasing their noise uniformly. The mismatch between odd and even channels is attributed to electric field coupling between the converter and the bonding wires that tie the strips to the ASIC inputs: these bonds are arranged in a double layer stack, those placed above being exposed to the 

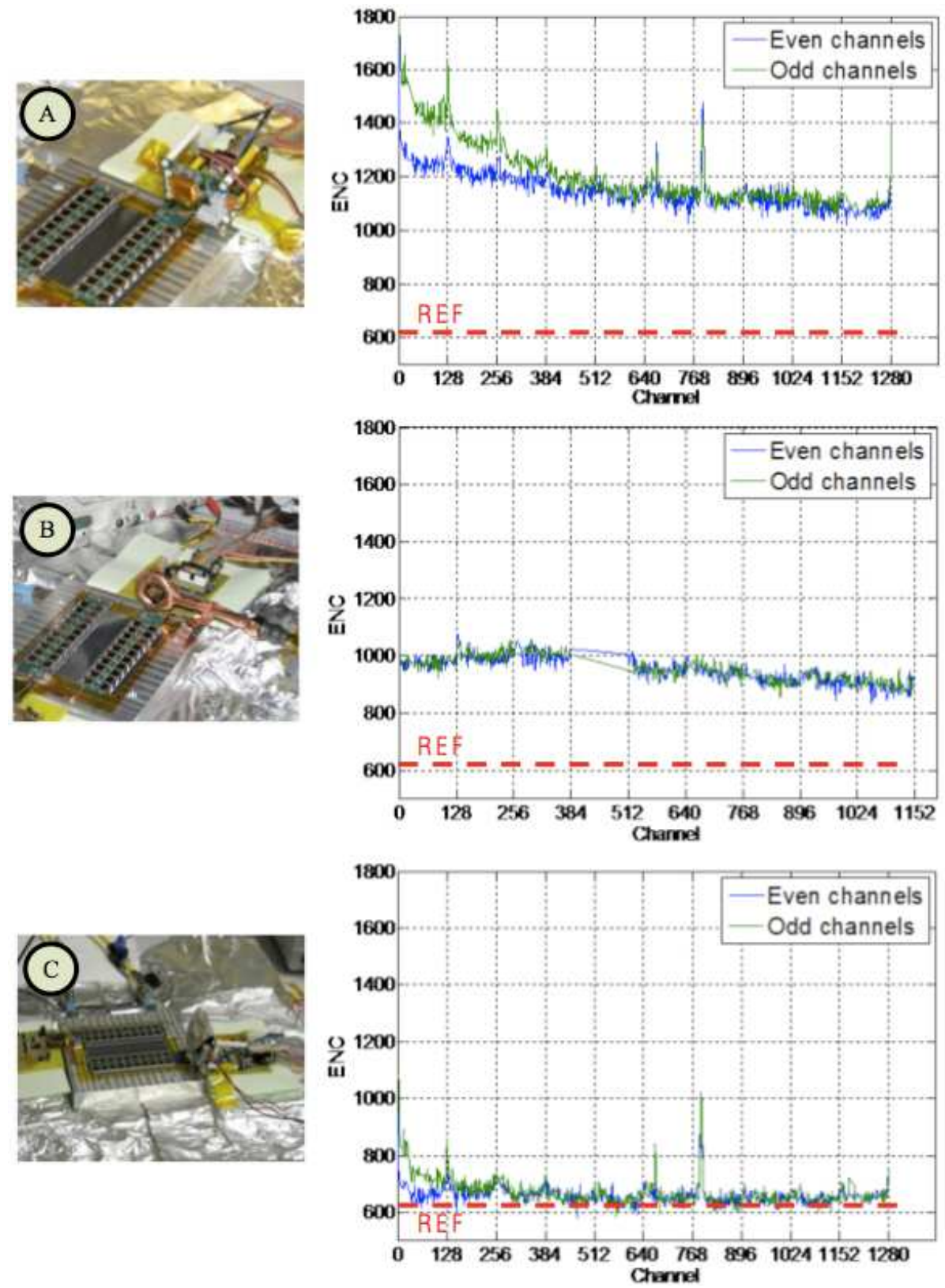

Figure 7. ENC susceptibility of the Liverpool front-end system against noise radiated by the converter (A), against magnetic field only (B) and using a shielded converter (C), compared with the reference ENC (REF line).

electric field coupling, and those sitting under being shielded by them. Wrapping the converter with a tinned copper foil helped to bring the noise down to the reference level (figure 7).

\subsection{Compatibility of the AMIS2 and SM01B converters with an ATLAS tracker module}

The compatibility of the AMIS2 and SM01B converters with the setups of the University of Geneva was explored, which showed susceptibility properties with Proto5 very similar to those observed 

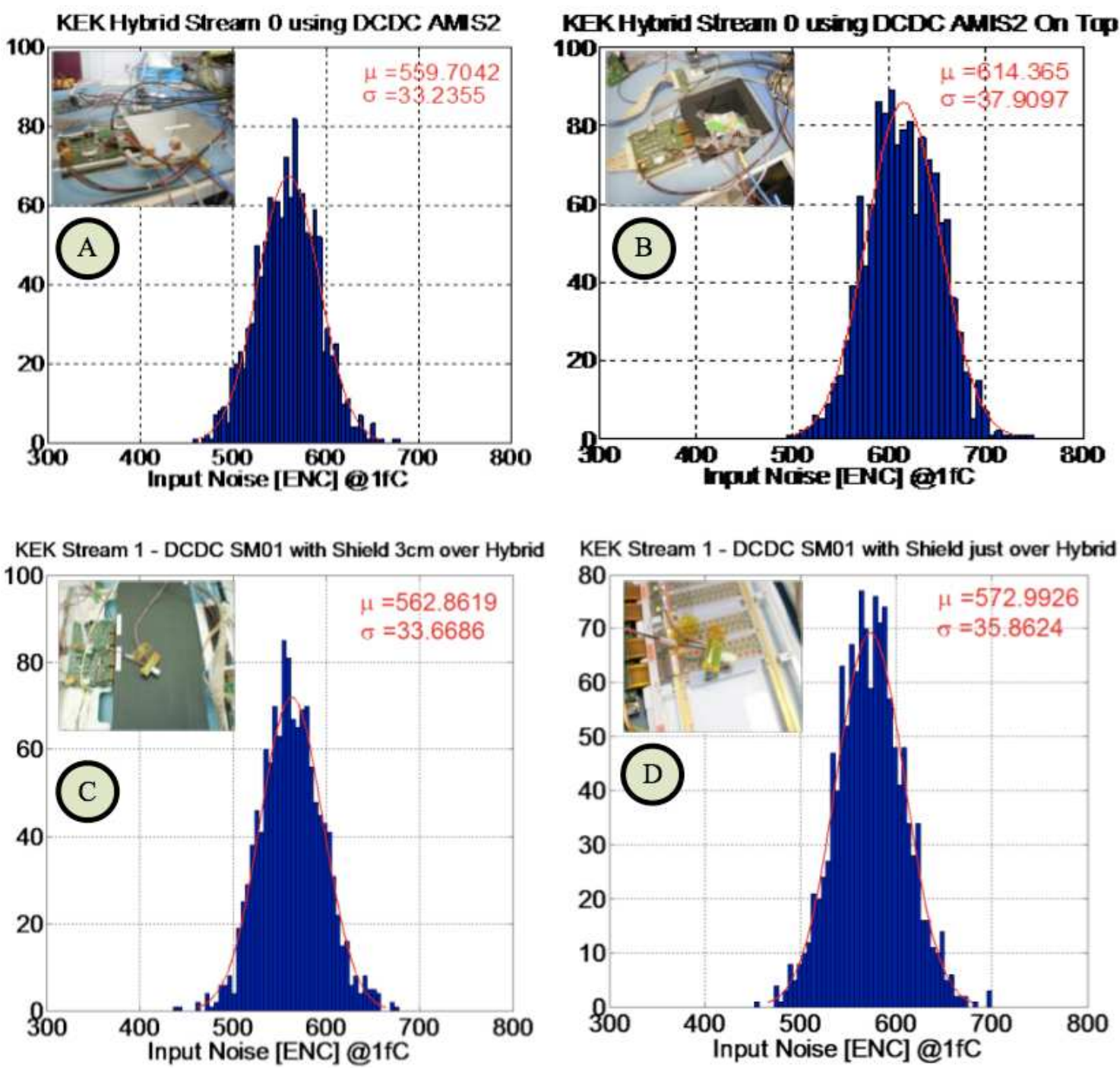

Figure 8. Noise susceptibility measurements of the University of Geneva tracker modules using the AMIS2_EMC converters (figures A and B) and with the SM01B converter (figures C and D) showing a noise increase of $10 \%$ and $2 \%$ respectively, when the converters are located at the most sensitive area of the hybrids.

on the Liverpool setup. The AMIS2-EMC converter was evaluated with a system made of a single module (figure 8): this system used separated power inputs for analog and digital circuitry, splitting the currents in $1 \mathrm{~A}$ and $3 \mathrm{~A}$ respectively, each being powered with one converter. Although the AMIS2-EMC board targets a nominal current of $2 \mathrm{~A}$, it was successfully used up to $3 \mathrm{~A}$ enabling the test on that system. Placing the two converters straight on top of the front-end ASICs being characterized for this test, facing down to maximize the coupling, a noise increase of only $10 \%$ was observed (ENC of 614 electrons instead of the 560 electrons reference level). This noise increase is comparable with the expected noise increase attributed to radiation damage in the detector and front-end ASICs, and is therefore acceptable.

Later on the University of Geneva developed a new system based on two modules instead 
of one, which used a common input power for both analog and digital sections of the system: it therefore required more than $4 \mathrm{~A}$ at its input. The analog voltage is produced within the $\mathrm{ABCN}$ ASICs using an embedded linear regulator. The large current required imposed the use of the SM01B board instead of the AMIS2-EMC board. Having only one converter and the front end regulator enabled, placing the converter facing down to the ASICs a noise increase of less than $2 \%$ was recorded (ENC of 573 electrons instead of the 563 electrons reference level, figure 8). A difference in the distance between the converter and the ASIC of $2 \mathrm{~mm}$ or $3 \mathrm{~mm}$ between the two setups is not excluded; it is however in both cases an excellent result that confirms the compatibility of the DC to DC converters with the two existing systems.

\section{Conclusions}

The compatibility between the converter modules and the front-end systems is a major concern to enable the use of DC to DC converters in upcoming HEP experiments. The existing systems are still using ASICs in $0.25 \mu \mathrm{m}$ CMOS technology, which requires larger currents than those targeted by the DCDC ASICs. Two types of converters have then been produced: those using commercial controllers able to deliver up to 5A, and those using the AMIS2 chip, limited to 2A. The noise generation and coupling mechanisms are now well controlled, and this enabled a strong optimization of the noise emissions finally implemented in the AMIS2-EMC module. Adding a shield cancels almost entirely the remaining radiated and conducted noise, achieving acceptable noise levels in the tested front-end systems even under extreme operation conditions. The commercial chip counterpart of the AMIS2-EMC board, namely the SM01B board, also fitted with a shield was found to be fully compatible on the Geneva system, also under extreme conditions.

Finally, the converters must be designed such that they can be industrially produced. The focus is now on the so far hand-made components of the converters. In collaboration with industry, a custom air core toroidal coil has been designed and prototypes have been produced with very good performances. Similarly, the manufacturing of the shield is now the object of several studies.

Most of the critical issues have now been solved: radiation tolerance, ASIC design, mitigation of noise couplings and production of custom components. All together, the production of converters samples is now possible enabling the systems tests at a larger scale with front-end prototypes currently under development.

\section{References}

[1] M. Weber et al., Performance and comparison of custom serial powering regulators and architectures for SLHC silicon trackers, in Proceedings of the Topical Workshop on Electronics for Particle Physics TWEPP-09, Paris, France, CERN-2009-006.

[2] C. Fuentes et al., Power distribution with custom DC-DC converters for sLHC trackers, Nucl. Sci. Symp. Med. Imag. Conf. (2009) 1300, Orlando, Florida U.S.A., October 2009.

[3] S. Buso, G. Spiazzi, F. Faccio and S. Michelis, Comparison of dc-dc converter topologies for future sLHC experiments, in Proceedings of the IEEE Energy Conversion Congress and Exposition, ECCE 2009, San Jose, California, September 2009. 
[4] S. Gonzalez-Sevilla et al., A silicon strip module for the ATLAS inner detector upgrade in the super LHC collider, Nucl. Instrum. Meth. (in press).

[5] A. Greenall, Prototype flex hybrid and module designs for the ATLAS Inner Detector Upgrade utilising the ABCN-25 readout chip and Hamamatsu large area Silicon sensors, in Topical Workshop on Electronics for Particle Physics TWEPP-09, Paris, France.

[6] B. Allongue, G. Blanchot, F. Faccio, C. Fuentes, S. Michelis and S. Orlandi, System Integration Issues of DC to DC converters in the sLHC Trackers, in Proceedings of the Topical Workshop on Electronics for Particle Physics TWEPP-09, Paris, France, CERN-2009-006.

[7] F. Anghinolfi et al., Performance of the ABCN-25 readout chip for the ATLAS Inner Detector Upgrade, in Proceedings of the Topical Workshop on Electronics for Particle Physics TWEPP-09, Paris, France, CERN-2009-006.

[8] F. Faccio et al., TID and displacement damage effects in vertical and lateral power MOSFETs for integrated DC-DC converters, IEEE Trans. Nucl. Sci. 57 (2009) 1790.

[9] F. Faccio et al., Development of custom radiation-tolerant DCDC converter ASICs, in Topical Workshop on Electronics for Particle Physics 2010, 20-24 September 2010, Aachen, Germany.

[10] B. Allongue, G. Blanchot, F. Faccio, C. Fuentes, S. Michelis and M. Perez, Characterization of the noise properties of DC to DC converters for the SLHC, in Proceedings of the Topical Workshop on Electronics for Particle Physics TWEPP-08, Naxos, Greece, 15 - 19 Sep 2008, pp.406-410. 